\title{
Extreme Light Infrastructure - Nuclear Physics (ELI-NP) European Research Centre
}

\author{
N.V. Zamfir ${ }^{\mathrm{a}}$ \\ ELI-NP, "Horia Hulubei" National Institute of Physics and Nuclear Engineering, Bucharest-Magurele, \\ Romania
}

\begin{abstract}
Extreme Light Infrastructure (ELI) is one of the 48 infrastructures of the European Strategic Forum for Research Infrastructure (ESFRI) roadmap and represents a major step forward in quest for producing extreme electromagnetic fields. Extreme Light Infrastructure - Nuclear Physics (ELI-NP) is one of the three pillars of the ELI initiative, which aims to use extreme electromagnetic fields for nuclear physics research. A high power laser system and a very brilliant gamma beam are the two main research equipment at the core of ELI-NP. Their targeted operational parameters are described. The related experimental areas are also presented, together with the main directions of the proposed scientific programme.
\end{abstract}

\section{Introduction}

Extreme Light Infrastructure (ELI) Pan-European initiative started in 2006 by a proposal on the European Strategic Forum for Research Infrastructure (ESFRI) roadmap. In 2007-2010 the idea was funded through European Union FP7 Programme by a preparatory phase project (ELI-PP) that had the mandate to decide the sites to host the infrastructure and the directory lines for the project. ELI will be a pan-European distributed facility with three research pillars: laser driven secondary radiation sources in Czech Republic, attosecond laser science in Szeged, Hungary and nuclear science in BucharestMagurele, Romania [1]. ELI is the first ESFRI Project financed by European Regional Development Fund.

The Extreme Light Infrastructure - Nuclear Physics (ELI-NP) in Romania will be a European research centre for ultra-high intensity lasers, laser matter interaction, nuclear science and material science using laser driven radiation beams. The laser intensities at ELI-NP will be in the range of $10^{23} \mathrm{~W} / \mathrm{cm}^{2}$. This multidisciplinary facility will provide completely new opportunities to study fundamental processes that occur in ultraintense laser fields during light-matter interaction.

The ELI-NP research centre will be located in Magurele, a town few kilometres away from Bucharest, Romania. ELI-NP will host two 10PW lasers and a Gamma beam system producing gamma beams with parameters beyond those available at the present state of the art machines. The total cost of the facility will be 293 million euro, without VAT. In September 2012 the project was approved by the European Commission and the first phase was financed with 180 million euro, with $83 \%$ financial support from the existing Structural Funds cycle (2007-2013) and 17\% from the Romanian National

\footnotetext{
${ }^{a}$ Corresponding author: victor.zamfir@eli-np.ro
} 
Budget. The second phase of financing will be granted in the next Structural Funds cycle (2014-2020). The entire facility will be operational in 2018.

This paper presents the planned high power laser system and the gamma beam system parameters. It also gives a glimpse of the scientific program associated with the ELI-NP facility $[2,3]$.

\section{ELI-NP Buildings Complex and Major Equipment}

The ELI-NP infrastructure will consists of a High Power laser system and a High Intensity Gamma Beam system. The latter will produce a highly collimated beam of radiation in the gamma ray domain, through the Compton backscattering of a laser beam off a beam of accelerated electrons produced by a linear accelerator. Both systems will be hosted by a building complex shown in Figure 1. The construction of the building complex started in May 2013 and will be completed in the spring of 2015.

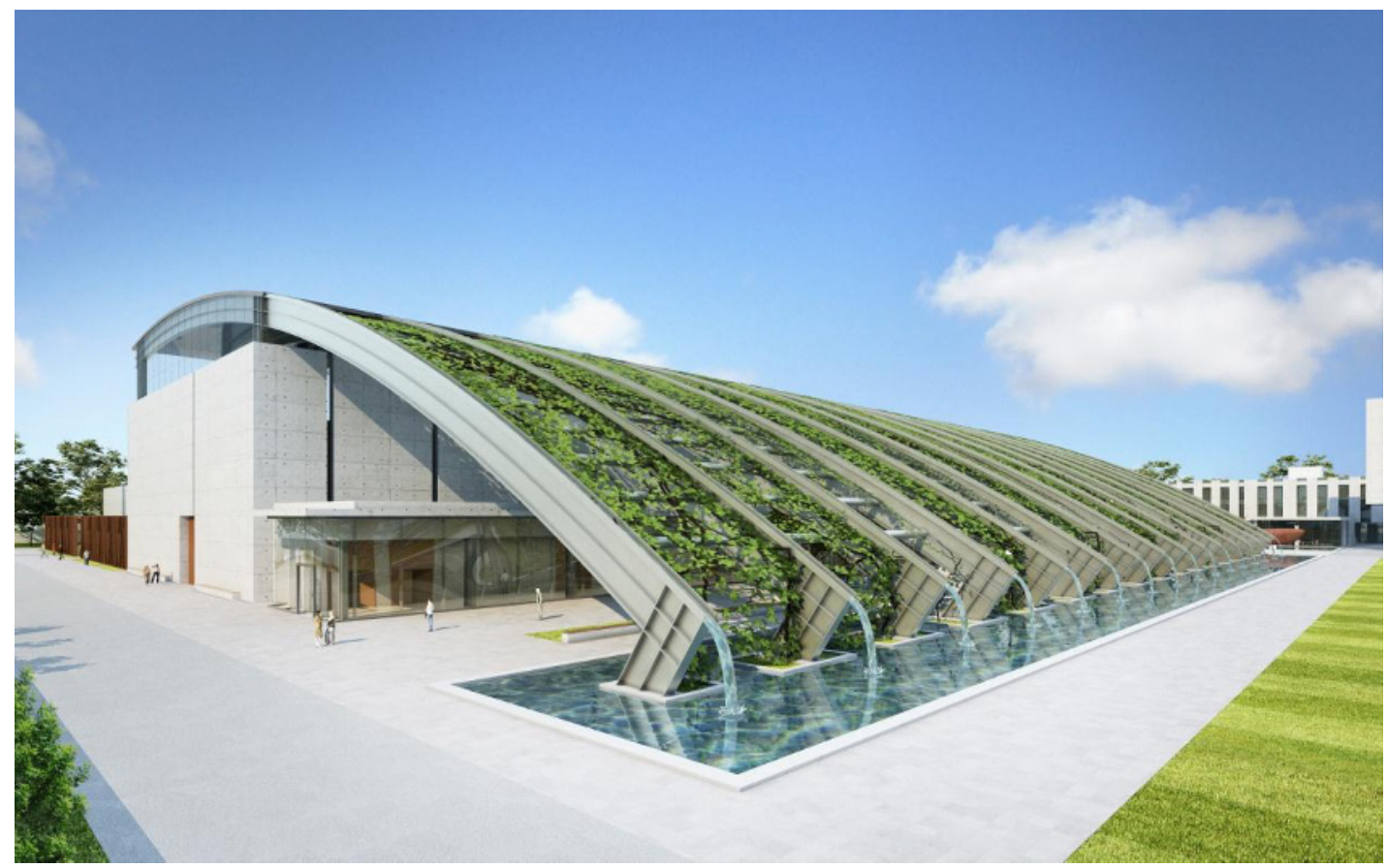

Figure 1. Architect's vision of the main buildings complex at ELI-NP: laser building, accelerator and experiments building, laboratories building.

The layout of the ELI-NP facility, covering an area of more than $10000 \mathrm{~m}^{2}$, is shown in Figure 2. It consists of the Laser hall (upper left side of the figure), Gamma Beam System Hall (lower part of the figure), Experimental Areas (middle) and Auxiliary Laboratories (upper right hand side of the figure). The high spatial accuracy of the beams requires a special design of the concrete base plate of the building to prevent vibrations. The state of the art equipment will require special clean-room, temperature and humidity conditions. The large amount of radioprotection concrete shielding is designed in a modular way to accommodate the planned experiments with a maximum of flexibility. 


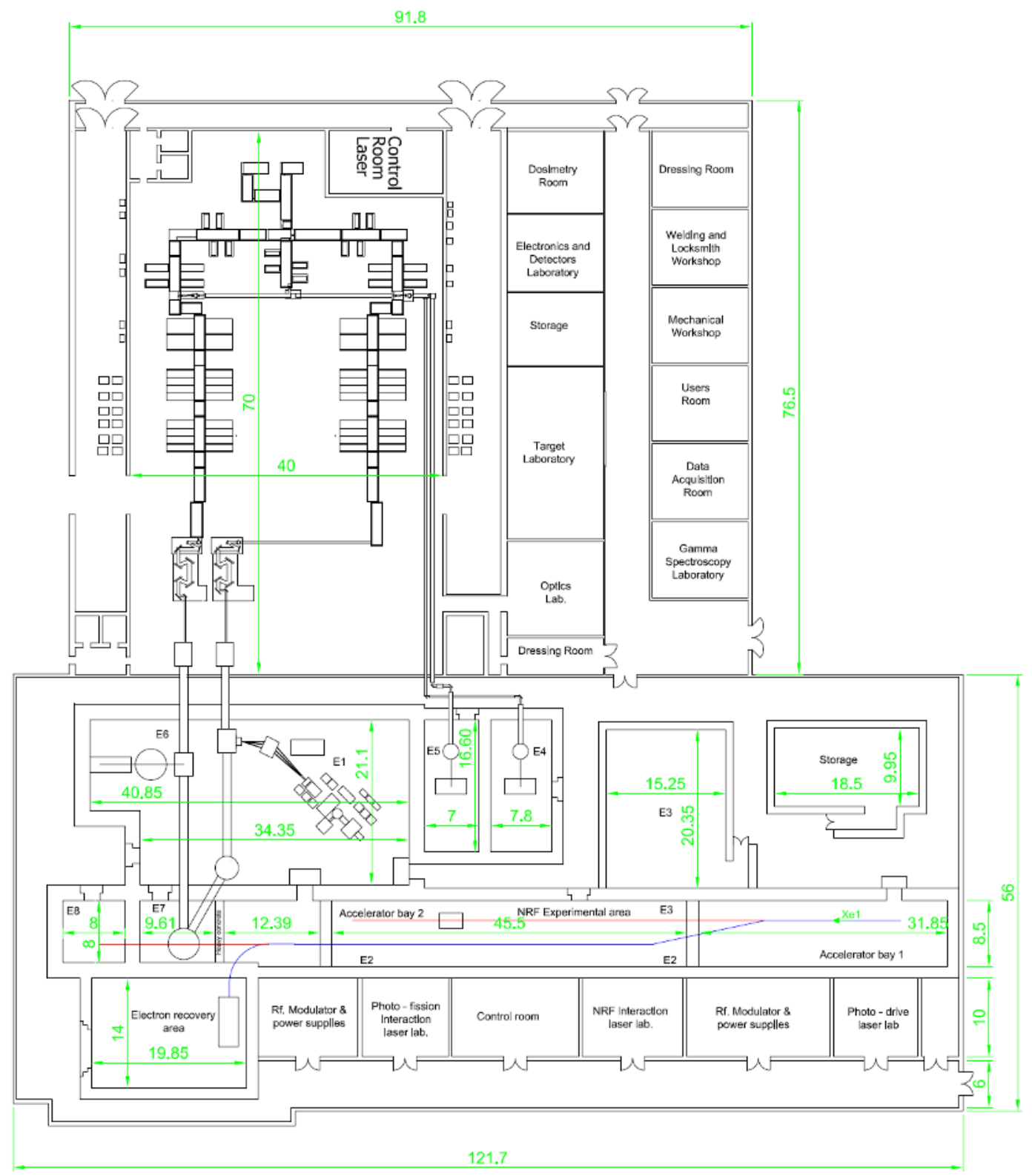

Figure 2. Layout of the ELI-NP facility. The dimensions are in meters

The high power laser system (HPLS) is a dual front-end system with two parallel amplification arms, each of them with three outputs: $100 \mathrm{TW}(\geq 10 \mathrm{~Hz}), 1 \mathrm{PW}(\geq 1 \mathrm{~Hz}), 10 \mathrm{PW}(\geq 1 / 60 \mathrm{~Hz})$. Each output will have its optical pulse compressor. The duration of the pulses from each of the 6 outputs of the HPLS shall be tunable from the best compression level to at least 5 ps pulse duration, with both positive and negative chirp.

The HPLS outputs will be synchronized with accuracy below $200 \mathrm{fs}$. In the long run, coherent combination of the outputs with the same output power levels is envisaged. The laser system will deliver pulses synchronously with the Gamma Beam System electron and gamma bunches. The 
synchronization system will be implemented at the HPLS front-end level. The pulse duration was chosen to be below $50 \mathrm{fs}$. A small focal spot is essential for experiments, in order to reach highest intensities on the target. Temporal contrast of the pulses, i.e. the ratio between the amplified spontaneous emission nanosecond background or satellite pulses and the main pulse, is a critical parameter at the very high field intensities to be generated on targets. The specified temporal contrast at the $0.1 \mathrm{PW}$ output level is specified to be in the range of $10^{11}: 1$, for both nanosecond and picosecond temporal range.

The HPLS will be constructed and commissioned by Thales Optronique S.A.S. France in association with S.C. Thales Systems Romania.

The ELI-NP Gamma Beam System (GBS) will enable the production of a brilliant, highly collimated beam of radiation in the gamma ray domain, through the Compton backscattering of a laser beam off a beam of accelerated electrons. This will ensure the possibility to tune with very high precision the energy of the gamma radiation produced in a range that is relevant for the scientific case of ELI-NP. Electrons will be accelerated up to $700 \mathrm{MeV}$ by a warm linac.

The key parameters of the GBS are presented in Table 1. The minimum output specifications for the Gamma beam were established in a series of workshops and meetings organized with the international scientific community interested in ELI-NP, in order to satisfy the need of the scientists for the progress of their research but also to have realistic expectancies that are technically feasible within the time frame of project implementation.

Table 1. Parameters for the gamma beam.

\begin{tabular}{|c|c|}
\hline Type & Range \\
\hline Photon energy & $0.2-19.5 \mathrm{MeV}$ \\
\hline Divergence & $\leq 2.0 \times 10^{-4} \mathrm{rad}$ \\
\hline Average Relative Bandwidth of Gamma-Ray Beam & $\leq 5.0 \times 10^{-3}$ \\
\hline Time-Average Spectral Density at Peak Energy & $\geq 5.0 \times 10^{3} \mathrm{ph} /(\mathrm{s} \mathrm{eV})$ \\
\hline Time-Average Brilliance at Peak Energy & $\geq 1.0 \times 10^{11} \mathrm{ph} /(\mathrm{s} \mathrm{mm} 2 \mathrm{mrad} 20.1 \% \mathrm{bw})$ \\
\hline Minimum Frequency of Gamma-Ray Macropulses & $\geq 100 \mathrm{~Hz}$ \\
\hline
\end{tabular}

\section{Experimental Programme}

The experimental programme at ELI-NP is based on the proposals reported in the White Book [2] and is summarized in Ref. [3].

ELI-NP will feature eight experimental rooms organized as shown in Figure 2:

- E1: laser induced nuclear reactions based on the 10PW laser beam;

- E2: nuclear resonance fluorescence and applications, based on low energy $(\sim 3 \mathrm{MeV}) \gamma$ beam;

- E3: positrons source;

- E4 / E5: accelerated particle beams induced by laser beams (0,1/1 PW) at high repetition rates;

- E6: intense electron and gamma beams induced by high power (10 PW) laser beams; 
- E7: experiments with combined laser (10 PW) and gamma beams;

- E8: nuclear reactions induced by high energy gamma beams (up to $19 \mathrm{MeV}$ ).

The technical experimental design reports (TDR) associated to each type of experiment are now in preparation. The TDRs corresponding to the use of the high power laser beams aim:

- to develop experiments using ion driven nuclear reactions where the ions are produced with the 10 PW laser. The most important scientific objective in this case is the study of neutronrich nuclei in the region of the waiting point $\mathrm{N}=126$. Such experiments require a huge experimental development in the field of laser driven ion acceleration according to new acceleration mechanisms, to be tested for the first time at ELI-NP.

- to develop ultra-relativistic electron sources, and subsequently, gamma beams for nuclear physics experiments. Complementary strong field quantum electrodynamics studies at the interaction between ultraintense laser pulses and solid targets were proposed.

- $\quad$ to make prospective studies based on the combination of HPLS 10PW beams with the GBS pulses. Thus, dynamics of the electrons in tightly focused, ultraintense laser fields, modifications of the vacuum constants and gamma beam assisted electron-positron production experiments were suggested in the ELI-NP White Book.

Another set of TDRs will be focused on using the GBS and will combine two main directions:

- Basic science - fundamental physics of perturbative and non-perturbative high-field quantum electrodynamics, high-resolution nuclear spectroscopy and astrophysics of the r-, sand p-processes in nucleosynthesis. The main achievements with the $\gamma$ beam facility are likely to occur as a result of the high resolution at higher nuclear excitation energies in studies related to collective modes in nuclei. For example, identifying the predicted neutron halo isomers closely below the neutron binding energy would open a new field of nuclear spectroscopy. Major advances are expected in the fields of photonuclear reactions related to nuclear astrophysics, as well as to photofission studies. The new extremely performing $\gamma$ beam opens new possibilities for high resolution spectroscopy at higher nuclear excitation energies. They will lead to a better understanding of nuclear structure at higher excitation energies with many doorway states, their damping widths, and chaotic behaviour, but also new fluctuating properties in the time and energy domain. The detailed investigation of the pygmy dipole resonance above and below the particle threshold is essential for nucleosynthesis in astrophysics.

- Applications - developing Nuclear Resonance Fluorescence (NRF) for nuclear materials and radioactive waste management, brilliant gamma, X-ray, neutron, positron and electron microbeams in material and life science, and techniques of laser acceleration and of a brilliant gamma beam. The project aims to develop techniques for remote characterization of nuclear materials or radioactive waste via NRF and this will be of large importance for the European society. The unsolved problems of long-term storage of radioactive waste from reactors, while having to deal with large amounts of old, insufficiently characterized radioactive waste, requires a precise isotopic characterization in the first place. The new production schemes of medical isotopes via the $(\gamma, n)$ reactions might also reach socioeconomical relevance. The investigation of new production schemes of medical isotopes (e.g., ${ }^{99} \mathrm{Mo}$ - currently used in therapies, ${ }^{195 \mathrm{~m}} \mathrm{Pt}$ - nuclear imaging to determine efficiency of chemotherapy, ${ }^{117 \mathrm{~m}} \mathrm{Sn}$ - emitter of low energy Auger electrons for tumor therapy) via $(\gamma, \mathrm{n})$ processes are also among the proposed areas of study of ELI-NP. Another relevant example of applied research is the investigation of new methods to produce thermal neutrons, through photonuclear reactions $(\gamma, n)$. These will be used in the study of bio-proteins, nanocomposites, fullerenes, and magnetic materials, to name a few. Another example would be the creation of an intense positron source by means of the $\left(\gamma, \mathrm{e}^{+} \mathrm{e}^{-}\right)$reaction, very useful in materials science. 
Finally, the last set of TDRs will focus on experiments induced by combined laser and gamma beams and among the experiments proposed by the scientific community are: Compton Scattering and Radiation Reaction of a Single Electron at High Intensities; Cascades of e+e- Pairs and Gamma Rays triggered by a Single Slow Electron in Strong Fields; The Real Part of the Index of Refraction of the Vacuum in High Fields: Vacuum Birefringence. ELI-NP will approach the field intensity boundary from which the observation of the first catalysed pair creation from the quantum vacuum should be achievable.

\section{Conclusions}

The ELI-NP facility will benefit from two major research equipment with beyond the state-of-the-art parameters, namely a high power laser system with two amplification arms to deliver $10 \mathrm{PW}$ and intensities on the target in the range of $10^{23} \mathrm{~W} / \mathrm{cm}^{2}$ at least every minute, and a gamma beam system to deliver up to $19 \mathrm{MeV}$ photons with extremely good brilliance and bandwidth. Their outstanding performances will allow for approaching a virgin science field, at the frontier between the strong field QED and nuclear physics.

\section{Acknowledgments}

The ELI-NP project is the result of an international collaborative effort of scientists from more than 20 countries and their contribution to the definition of the project is gratefully acknowledged.

The ELI-NP project is co-funded by The European Union through the European Regional Development Fund.

\section{References}

1. ELI - Extreme Light Infrastructure Science and Technology with Ultra-Intense Lasers Whitebook, Editors Gérard A. Mourou, Georg Korn, Wolfgang Sandner, John L. Collier, at THOSS Media GmbH, (2011)

2. ELI-NP White Book, http://www.eli-np.ro/documents/ELI-NP-WhiteBook.pdf

3. Dietrich Habs, Toshiki Tajima, Victor Zamfir, Extreme Light Infrastructure - Nuclear Physics (ELI-NP): New Horizons for Photon Physics in Europe, Nuclear Physics News vol.21,nr. 1 (2011) p.23-29 